\title{
Comprehensive Mobility Prediction Based Clustering Algorithm for Ad Hoc UAV Networks
}

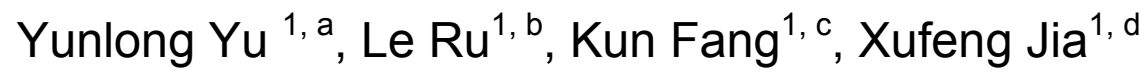 \\ ${ }^{1}$ School of Aeronautics and Astronautics Engineering College, Air Force Engineering University, \\ Xi'an 710038, China; \\ a yuyunlong426@126.com, ${ }^{b}$ rule@163.com, ㄷangkun@163.com, jijaxufeng@163.com
}

Keywords: ad hoc UAV networks, highly dynamic, large scale, comprehensive mobility prediction, clustering

\begin{abstract}
Clustering is an effective method which can increase the performance of large-scale ad hoc UAV networks. However, the ad hoc UAV networks have the feature of high mobility and quick network topology change, using conventional clustering algorithm will lead to the decrease of link connection lifetime and cluster head lifetime, frequent updates of cluster topology would cause the instability of cluster structure and the increase of control overhead. In order to solve the problem that traditional clustering algorithm cannot adapt to the highly dynamic large-scale ad hoc UAV networks, CMPC (Comprehensive Mobility Prediction Based Clustering) algorithm is proposed. This algorithm predicts the comprehensive relative mobility between two UAVs which can get from the signal feature of Hello packets. Making use of the comprehensive stability between two UAVs which derives from comprehensive mobility between two UAVs, we can conduct the cluster formation and maintenance effectively. Simulations have shown that the CMPC algorithm outperforms the classical clustering algorithm in terms of average link connection lifetime and average cluster head lifetime, which can make the cluster structure more stable. As a result, this algorithm is ideal for highly dynamic large-scale ad hoc UAV networks.
\end{abstract}

\section{Introduction}

The recent years have witnessed a wider application of UAVs, covering many fields for military and civilian purpose. UAVs also play a significant role in modern warfare, implementing a variety of missions including reconnaissance, surveillance, information gathering, communication relay, fast attacking. The modern warfare is characterized for fierce conflicting, wide coverage, large amount of information, and a single UAV would be unable to meet the demands of it, thus multi-UAVs cooperative combat become a focus of current researches. Flight formations are usually designed for multi-UAVs to implement missions cooperatively.

An ad hoc UAV network can be applied to interchange data concerning mission planning, flight status and information when multi-UAVs performing tasks in flight formation, which improves the situation awareness of the UAV formations. When facing a wide range of battlefield environment, many UAV formations are needed to carry out tasks, as a result, the number of UAVs are increasing dramatically. If using traditional methods maintain the large-scale ad hoc UAV networks, it may cause the degradation of performance. Clustering is an efficient network management method, and the hierarchical network structure obtained using the clustering algorithm can improve the performance of ad hoc UAV networks, and reduce the complexity of network management. Therefore, clustering algorithm is ideal for large-scale ad hoc UAV networks.

However, ad hoc UAV network has the feature of greater node mobility, faster network topology variance than mobile ad hoc network. As a result, the links between UAVs are very instability. The mobility-aware clustering algorithm uses node's moving characteristic to conduct the cluster formation and maintenance, the main idea is to group mobile entities with similar speeds into the same cluster, which can establish stable links between intra-cluster nodes. Thus, the phenomenon of rebuilding the links and clusters is decreased. 
A well-known example of mobility-based clustering algorithm is MOBIC [1], where the relative mobility is estimated by two successively received packets' power. But it does not calculate the distance and relative speed accurately. Therefore, it can only apply to the scenario which node's speed and direction are roughly same, such as the highway. If the node's speed and direction change frequently, the performance of MOBIC may be degraded. CEMCA [2] considers node's connectivity, residual power, and node's mobility. The cluster head election is based on a weighted sum of the three different parameters. This method can adapt to different scenarios through adjusting weight. However, it cannot fulfill the highly dynamic ad hoc UAV networks because its method of handling node's mobility is not perfect. The methods in [3] and [4] derive from MOBIC and CEMCA, which cannot adapt to highly dynamic networks. MPCR [5] is specially designed for ad hoc UAV networks. Its clustering algorithm uses location information to calculate the predicted value of link connection lifetime between two UAVs, and then gets link connectivity probability. The UAV with the largest sum of link connectivity probability to its one-hop neighbors is chosen as the cluster head. MPCA [6] and the algorithm proposed in [7] are almost the same as MPCR, which use the location information to predict the link expiration time. The algorithm proposed in [8] brings the location information into Gauss-Markov model, and then predict the mobility of nodes. The location information derives from GPS, so MPCR and the algorithm proposed in [8] will introduce location error of GPS and external disturbance, which making the moving parameter inaccurate and then causing those algorithms invalid. In [9], the authors proposed a learning automata- based weighted cluster formation algorithm (MCFA), MCFA can adapt to highly dynamic ad hoc UAV networks whose direction of motion and mobility speed are random variables with unknown distributions. However, the control message overhead will become large when the speed of nodes is too fast. In the Mobility Adaptive Clustering Algorithm (MACA) [10], a sensor node selects itself as a cluster head based on a Single Point Predictor for the combined criterion prediction. But the MACA is only suitable for wireless sensor networks whose mobility is very weak.

We will utilize the signal characteristics of received Hello packets to predict the mobility of UAV. This method can be independent of the GPS, and it can take good advantage of Hello packets. SECA [11] considers the signal strength as an important criterion of clustering, but signal strength cannot predict the mobility of nodes completely. MPBC [12] calculates the relative moving speed by collecting the signal strength of Hello packets and Doppler shift. The node with the minimum sum of relative speed to its neighbors is chosen as the cluster head. However, the process of formula derivation in [12] uses approximate treatment, which can make speed calculation inaccurate. DDVC [13] calculates the relative radial moving speed by Hello packets' Doppler shift, and then get a new metric called the Doppler value(DV), which can be used to form stable clusters and also for cluster maintenance. However, the DDVC algorithm is specially designed for pseudolinear highly mobile ad hoc networks. A pseudolinear mobile entity moves in a relatively linear path without frequently changing its direction and speed.

We propose a model which can predict the mobility of UAV comprehensively, as a result, CMPC (Comprehensive Mobility Prediction Clustering) is proposed. The output value of the model can provide cluster formation and maintenance with exact gist.

\section{Mobility Model}

In the face of a wide range of battlefield environment, there usually have many UAV formations. Figure 1 shows several common forms in process of formation moving, A represents a formation merger; B represents a normal formation; $\mathrm{C}$ represents a formation partition. It also includes the formation generation and members regulation. Therefore, we should propose a mobility model which can imitate highly dynamic multi-UAVs' mobile scenario. 


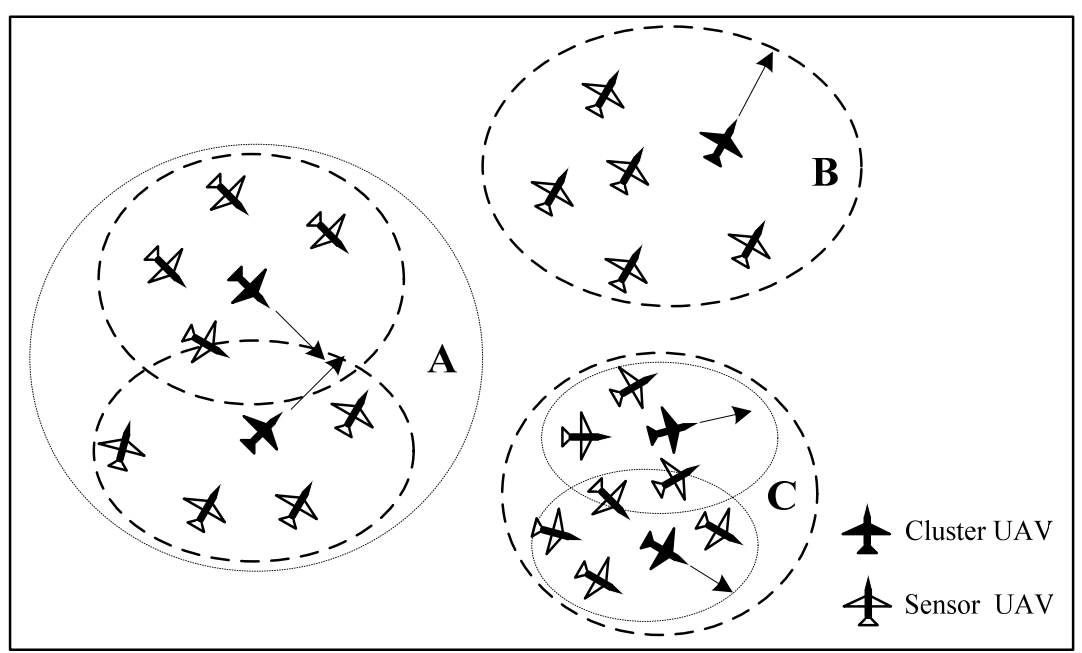

Fig. 1 The common forms in process of formation moving

The mobility model proposed in [14] includes the formation partition and merger, but it still needs to be improved. We propose a new mobility model based on the RRGM model [14], which can imitate highly dynamic large-scale multi-UAVs' mobile scenario.

$<1>$ The initialization of UAV formation. All UAVs are deployed randomly in the simulation region, at the same time, several disjoint circles whose radius is equal to communication range of UAV are deployed randomly in the simulation region. We define that the UAVs in the same circle form a formation, the UAV that does not locate in any one of the circles will join the closest formation.

$<2>$ The initialization of mission region and target. A number of mission regions are randomly distributed in the simulation region. A number of targets are randomly distributed in each mission region.

$<3>$ Mission region and target assignment. In this mobility model, we assume that all UAVs are carrying out reconnaissance task synergistically, the key point is how to assign different targets to different UAVs. In this paper, we simplify the problem of mission region and target assignment. We assume that the assignment is already completed. All mission regions are assigned to different UAV formations randomly, and we must guarantee that each mission region is reconnoitered by only one UAV formation. When some UAV formation has already reconnoitered the mission regions assigned to it, a new mission region is generated. The generating of a new mission region represents the change of hot spot in the battlefield. When an UAV formation is about to enter into some mission region, all the targets in this mission region are assigned to different UAVs in this formation randomly. We also guarantee that each target is reconnoitered by only one UAV. If the UAV formation has already reconnoitered the current mission region, the UAV formation will rush to next mission region.

$<4>$ The movement of the UAV formation. Each UAV formation has some mission regions to reconnoiter, and each UAV in the UAV formation has some targets in some mission regions to reconnoiter. Each UAV formation moves to its first mission region, the detailed process is as follows. When the UAV formation is close to the first mission region, the UAVs in the UAV formation move to their respective targets in the first mission region at varying speeds. When an UAV arrives at the first target and completes the reconnaissance task, it continues to move to the next target. If this UAV arrives at the final target, it will move randomly around the target and wait the other UAVs in this formation arriving at their final targets. When all the UAVs in this formation complete the reconnaissance task of its first mission region, this formation moves to next mission region and repeat the above-mentioned process.

$<5>$ Formation merger. At a fixed time interval, two UAV formations whose distance is nearest merge into a new bigger formation. The new UAV formation selects the mission regions which are not completed by some formation of the former two formations as its mission regions. Finally, this new formation repeats the detailed process proposed in $\langle 4>$. If the selected UAV formation is 
carrying out reconnaissance task of some mission region, the formation merger will be proceeded after the formatin completing reconnaissance task of the mission region.

$<6>$ Formation partition. At a fixed time interval, the UAV formation which is selected randomly splits into two smaller clusters. One formation selects the mission regions which belongs to the former formation as its mission regions. Some new mission regions are assigned to another formation, these mission regions derive from the mission regions which is discarded by the process of formation merger. Finally, the two new formations repeat the detailed process proposed in $\langle 4\rangle$. At the same time, if the selected UAV formation is carrying out reconnaissance task of some mission region, the formation partition will be proceeded after the formatin completing reconnaissance task of the mission region.

\section{Comprehensive Mobility Prediction}

We can predict the relative mobility between two UAVs comprehensively through two indexes. One is the movement stability between two neighboring UAVs. Another is the link subsistence probability between two neighboring UAVs.

\section{The movement stability between two neighboring UAVs}

According to the free space attenuation model [15], the received signal strength $P_{i j}$ of the Hello packets in current $\mathrm{UAV} \mathrm{i}$ transmitted from the neighboring $\mathrm{UAV} \mathrm{j}$ in a distance $\mathrm{d}$ is

$$
P_{i j}=P_{t} G_{t} G_{r}\left(\frac{\lambda}{4 \pi d}\right)^{2}
$$

where $\lambda$ is the length of radio wave, $G_{r}$ is the receiving antenna gain, $G_{t}$ is the transmitting antenna gain, $P_{t}$ is the transmitting power of Hello packets from neighboring UAV.

We assume that the covering range of antenna is a circular region whose radius is $\mathrm{R}$. Hence the threshold value of received signal strength of Hello packets from neighboring UAV is defined as:

$$
P_{\text {threshold }}=P_{t} G_{t} G_{r}\left(\frac{\lambda}{4 \pi \mathrm{R}}\right)^{2}
$$

The threshold value of received signal strength can be calculated based on the known information of current UAV and the information included in the Hello packets transmitted from neighboring UAV. Using the threshold value of received signal strength and the real received signal strength, we have

$$
X_{i j}\left(t_{k}\right)= \begin{cases}0 & P_{i j} \leq P_{\text {threshold }} \\ 1-\frac{P_{\text {threshold }}}{P_{i j}} & P_{i j} \geq P_{\text {threshold }}\end{cases}
$$

The Bienayme-Chebyshev inequality [16] is as follows:

$$
P\{|X-E(X)|<\varepsilon\} \geq 1-\frac{\operatorname{var}(X)}{\varepsilon^{2}}
$$

where $\mathrm{X}$ is a discrete variable, $\mathrm{E}(\mathrm{X})$ is the expectation value of $\mathrm{X}, \operatorname{var}(\mathrm{X})$ is the variance of $\mathrm{X}, \varepsilon$ is an arbitrary positive number.

If $\operatorname{var}(X)=0$, then $P\{|X-E(X)|<\varepsilon\}=1$, which means the variable $\mathrm{X}$ is equal to its expected value, which also means that the smaller the variance of $X$ is, the closer of $X$ would be to its expected value, and the smaller the varied quantity of $\mathrm{X}$ would be.

According to multiple measurements of $\mathrm{X}$, the variance $\operatorname{var}(X)$ is:

$$
\operatorname{var}(X)=\left(\sum_{k} \frac{X_{k}^{2}}{n}\right)-\left(\sum_{k} \frac{X_{k}}{n}\right)^{2}
$$

Take the values of $X_{i j}\left(t_{k}\right)$ at different moments as the multiple measured values of $\mathrm{X}$ and substitute them into the equation (5), and we can get the variation of the received signal strength of the Hello packets in current UAV i transmitted from the neighboring UAV $j$, and then we can judge 
the movement condition of neighboring UAV j relative to the current UAV $i$. The movement stability between two neighboring UAVs $N S_{i j}$ is calculated as follows:

$$
\begin{gathered}
\operatorname{var}\left(X_{i j}\right)=\left(\sum_{k} \frac{X_{i j}\left(t_{k}\right)^{2}}{n}\right)-\left(\sum_{k} \frac{X_{i j}\left(t_{k}\right)}{n}\right)^{2} \\
N S_{i j}=1-\operatorname{var}\left(X_{i j}\right)
\end{gathered}
$$

The larger the value of $N S_{i j}$ is, the better the movement stability between two neighboring UAVs is.

\section{The link subsistence probability between two neighboring UAVs}

In ad hoc UAV networks, each UAV broadcasts Hello packets periodically. We can calculate the link subsistence probability between current UAV i and neighboring UAV $j$ through extracting the characteristic parameters from the received signal. The parameters which we need to extract from the received signal include the frequency of signal and the strength of signal. On the basis of literature [12], there exists two mobile scenarios: one is approaching scenario, the other is receding scenario.

Figure 2 represents the approaching scenario, we assume that UAV i is static and located at a, $\mathrm{UAV} \mathrm{j}$ moves from $\mathrm{b}$ to $\mathrm{c}$, and its relative movement speed is $v_{r}$. Dashed circle whose center is a represents the maximum communication range of UAV i. The link interruption happens under the condition that the two UAVs are at their maximum communication range.

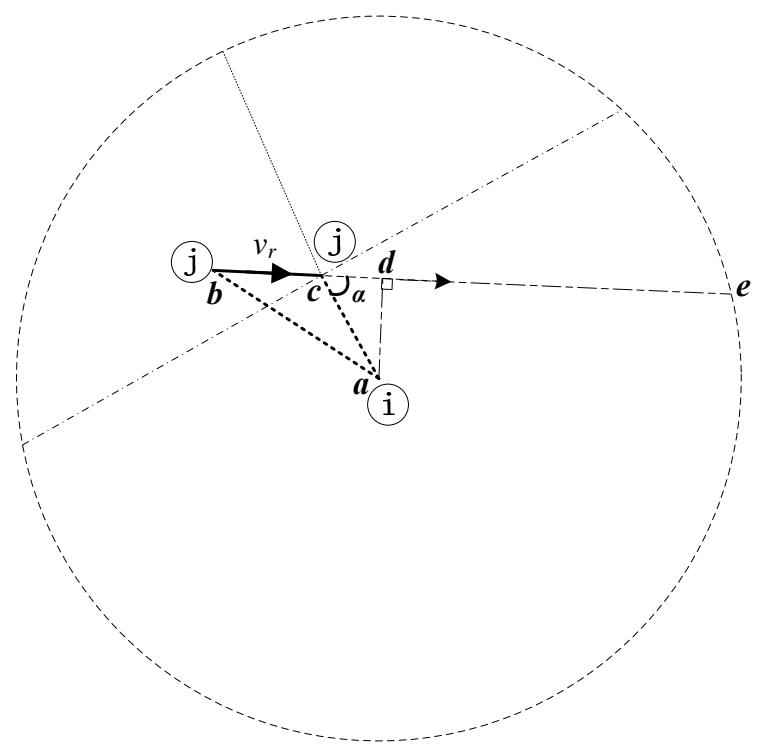

Fig. 2 The "approaching" scenario

The link subsistence probability between UAV i and UAV $\mathrm{j}$ has something to do with the relative movement speed and the distance between two UAVs. Therefore, the link subsistence probability can be calculated as follows:

$$
L S P_{i j}=\left\{\begin{array}{cl}
\frac{d_{c e}}{v_{r} t_{\text {threshold }}}, & \frac{d_{c e}}{v_{r}}<t_{\text {threshold }} \\
1, & \frac{d_{c e}}{v_{r}} \geq t_{\text {threshold }}
\end{array}\right.
$$

where $L S P_{i j}$ represents the link subsistence probability between UAV i and UAV $\mathrm{j}$. The larger the value of $L S P_{i j}$ is, the better the link is. $d_{c e}$ represents the distance between c and e. $t_{\text {threshold }}$ represents the threshold value of the link subsistence prediction lifetime. When the relative speed between two 
neighboring UAVs is in close to zero, $\frac{d_{c e}}{v_{r}}$ goes to infinity. We consider $\frac{d_{c e}}{v_{r}}=t_{\text {threshold }}$ when $\frac{d_{c e}}{v_{r}} \geq t_{\text {threshold }}$, therefore $L S P_{i j}=1$.

$d_{c e}$ and $v_{r}$ are calculated by the characteristic parameters of received signal. The detailed method of calculation refers the method which is mentioned in [12], but we should replace the approximate calculation by accurate calculation. The calculation of the link subsistence probability in receding scenario uses the same method that we use in the approaching scenario.

\section{Comprehensive mobility prediction based clustering algorithm}

We propose a clustering algorithm which can apply to highly dynamic large-scale ad hoc UAV networks. This algorithm includes cluster formation and cluster maintenance. When the UAV network is first established, the cluster formation is performed. The cluster maintenance is designed to solve problems caused by random high-speed movement of UAV, keep the stability of the cluster.

At first, the current UAV will calculate the sum of the value of $A_{i j}(t)$ to its one-hop neighbors in CMPC algorithm. However, in order to make sure the stability of the established cluster, not all one-hop neighboring UAVs of the current UAV are in the calculation range. In the process of calculation, we do not consider the neighboring UAVs which the value of $A_{i j}(t)$ is less than $A_{\min }$. In ad hoc UAV networks, each UAV will calculate the sum of the value of $A_{i j}(t)$ to its one-hop neighbors, which represents the probability of them becoming the cluster head. The process of calculation is as follows:

$$
\begin{gathered}
A_{i j}(t)=k_{1} \times L S P_{i j}+k_{2} \times N S_{i j} \\
C H P_{i}(t)=\sum_{j \in N} A_{i j}(t)
\end{gathered}
$$

where $A_{i j}(t)$ represents the comprehensive stability between UAV $\mathrm{i}$ and UAV $\mathrm{j}$ which derives from the comprehensive mobility between two UAVs. $\mathrm{CHP}_{i}(t)$ represents the probability of the current UAV i becoming the cluster head. The higher the value is, the more likely the current UAV i is to become a cluster head. $N$ is the set of one-hop neighboring UAVs of the current UAV i, but $N$ does not include the neighboring UAVs which the value of $A_{i j}(t)$ is less than $A_{\min }$.

\section{Cluster formation}

\section{The selection of cluster head}

When the ad hoc UAV network is first established, all the UAVs are in the orphan state. They will broadcast Hello packets periodically and build the neighboring list based on the Hello packets transmitted from other UAVs. The first step of cluster formation is the selection of cluster head. The process of selecting cluster head is as follows:

$<1>$ When the current UAV receives two successive Hello packets transmitted from the neighboring UAV, the current UAV calculates the link subsistence probability and the movement stability between the current UAV and the neighboring UAV through the section 2.1 and 2.2.

$<2>$ Each UAV calculates the probability of it becoming the cluster head through equation (10), and broadcasts the probability to its one-hop neighbors in the Hello packet. When an UAV i receives the $C H P_{j}(t)$ from its neighbors, it compares them with its own $C H P_{i}(t)$. If its $C H P_{i}(t)$ is larger than others' $C H P_{j}(t)$, the UAV i continues to broadcast the probability of it becoming the cluster head in the Hello packet. If its $C H P_{i}(t)$ is less than others' $C H P_{j}(t)$, the UAV i broadcasts the normal Hello packets which does not include the probability of it becoming the cluster head. 
$<3>$ If the UAV i do not receive others' $C H P_{j}(t)$ for a long time, the probability of the UAV i becoming the cluster head is the largest in the range of its one-hop. Therefore the UAV i becomes the cluster head and broadcasts an announcement in Hello packets.

\section{The selection of cluster member}

If the current UAV receives one cluster head announcement, and the comprehensive stability between the current UAV and the cluster head is larger than $A_{\text {min }}$, the current UAV joins the cluster.

If the current UAV receives more than one cluster head announcement, the current UAV selects the one that can provide it with the largest comprehensive stability as its cluster head.

\section{Cluster maintenance}

The highly dynamic situation of ad hoc UAV networks can reduce the stability of cluster structure, therefore, the rebuilding of clusters and links is very common in this network, which can increase the number of control messages considerably. CMPC algorithm can predict the breakage of links and the change of clusters, and then reduce the link rebuilding times and the cluster rebuilding times, which eventually decrease the number of control messages. In ad hoc UAV networks, there are four conditions in the cluster maintenance: cluster member leaves or joins some cluster, cluster head rotation, cluster merger and cluster partition.

\section{Cluster member leaves or joins some cluster}

Due to the high-speed movement of UAV and limited transmission range, an established association between a cluster member and its cluster head may be broken after the cluster formation stage. All cluster members in some cluster can be divided into two categories:

$<1>$ Some cluster members can not only receive the Hello packets transmitted from the current cluster head, but also can receive the Hello packets transmitted from neighboring cluster heads.

$<2>$ Some cluster members can only receive the Hello packets transmitted from the current cluster head.

Each cluster member in classification $<1>$ saves a specific neighbor list, which is used for storing the appropriate neighboring cluster heads and the comprehensive stability between the cluster member and neighboring cluster heads. If the comprehensive stability $A_{\text {im }}$ between the cluster member $\mathrm{i}$ and the current cluster head $\mathrm{m}$ is less than the comprehensive stability $A_{i j}$ between the cluster member $i$ and the neighboring cluster head $j$, the neighboring cluster head $j$ will be stored in the specific neighbor list of the cluster member $i$. If $A_{i j}<A_{i m}$, the cluster head $j$ will be removed from the specific neighbor list of the cluster member i. When one cluster member tends to keep away from the current cluster head, it starts to establish the specific neighbor list and updates the list periodically. If the comprehensive stability between the cluster member $\mathrm{i}$ and the current cluster head $\mathrm{m}$ is less than the minimum comprehensive stability $A_{\min }$, the link between the cluster member $\mathrm{i}$ and the current cluster head $\mathrm{m}$ will be broken. The cluster member i sends out a deassociation request to its current cluster head $\mathrm{m}$, the cluster member $\mathrm{i}$ then selects the cluster head with the largest comprehensive stability in the specific list and associates with it.

The reassociation time is defined as the time required for a cluster member to associate with another cluster head after it breaks the association with its current cluster head. The cluster member in CMPC algorithm can predict a possible association loss and reassociate to an appropriate cluster head before losing its current association. Therefore, the reassociation time in CMPC algorithm can be greatly reduced compared with that in conventional clustering algorithms.

Of course, the specific neighbor list of the cluster member in classification $<1>$ may be empty. When the association of the cluster member to its current cluster head is lost, the cluster member is reset to the orphan state and keeps broadcasting the Hello packets while waiting for the Hello packets from the other cluster heads. This method can be applied to the situation in classification $<2>$.

\section{Cluster head rotation}

The mobility of the UAVs in cluster changes with time, a current cluster head may no longer be appropriate for being a head with the time goes by. In CMPC algorithm, the current cluster head i is 
not appropriate for being a head when its value of $C H P_{i}(t)$ decreases drastically, and we need select a new cluster head for the cluster. When the cluster is established, the cluster head updates the value of $C H P_{i}(t)$ periodically according to the received Hello packets, and calculates the average value of $C H P_{i}(t)$ on the basis of the cluster's scale.

$$
\operatorname{ACHP}_{i}(t)=\frac{1}{M} \sum_{j \in N} A_{i j}(t)
$$

where $M$ represents the number of UAVs in the cluster.

When the decline between two values of $A C H P_{i}(t)$ is larger than $\phi$ with time interval $T$, it indicates that the current cluster head $i$ is no longer appropriate for being a head. Therefore, the UAV $\mathrm{i}$ gives up the status of cluster head and broadcasts a cluster head rotation announcement. All the members in this cluster enter the initial clustering phase, and a new cluster head will be elected.

$$
\triangle A C H P_{i}(n T)=A C H P_{i}(t-n T)-A C H P_{i}(t)>\phi
$$

where $T$ is the period of the Hello packets, $n$ is the positive integer.

\section{Cluster merger}

In ad hoc UAV networks, cluster merger means that two clusters merge into one cluster. The main feature is that a cluster head can receive the other cluster head's Hello packets. When two cluster heads move into each other's coverage area, the transmission collisions are very frequent, which reduces the performance of the network. Therefore, the cluster merger is necessary.

A general method for cluster merger is proposed in [17]. After comparing some specific metrics, one cluster head is degraded to a cluster member of the other cluster head, and the cluster members associated with the degraded cluster head then look for proper clusters to join in. Based on the aforementioned method, we propose a new method of cluster merger, which can adapt to ad hoc UAV networks. The process is as follows:

Cluster head i receives Hello packets from another cluster head $\mathrm{j}$, if this phenomenon lasts for a short time, the process of cluster merger will not be triggered. If cluster head i can receive Hello packets from another cluster head $\mathrm{j}$ for a long time and satisfy condition $\langle 1><2>$, the two clusters will merge into one cluster. At the same time, cluster head i inform all the members in this cluster and cluster head $\mathrm{j}$ of cluster merger.

$<1>$ The comprehensive stability $A_{i j}$ between cluster head $i$ and cluster head $\mathrm{j}$ is larger than $\lambda A_{\min }$.

$<2>$ There are more than half of cluster head j's members whose comprehensive stability to cluster head $i$ is larger than $\lambda A_{\text {min }}$.

Cluster head $\mathrm{i}$ and cluster head $\mathrm{j}$ calculate the value of $C H P(t)$ according to equation (10), the cluster head with the larger value of $C H P(t)$ is selected as the cluster head of the merged cluster. In the process of calculation, they just consider the UAVs in previous clusters by using the special Hello packets. Only the UAVs in previous clusters can join the merged cluster. This method decreases the ripple effect caused by the local re-clustering.

\section{Cluster partition}

In ad hoc UAV networks, cluster partition means that the cluster splits into two smaller clusters. When some cluster begins to split, the following phenomenon occurs. The comprehensive stability between the current cluster head and a part of cluster members remain unchanged basically, but the comprehensive stability between the current cluster head and another part of cluster members are declining. The number of the cluster members whose comprehensive stability are declining is equal or greater than $\alpha M$. The value of $M$ represents the number of UAVs in the cluster.

Set A includes the current cluster head and the cluster members whose comprehensive stability remain unchanged. Set B includes the cluster members whose comprehensive stability are declining. When the comprehensive stability between the cluster head and more than half of UAVs in set B are less than or equal to $A_{\min }$, we consider the current cluster does not exist, set $A$ and set $B$ have become two independent clusters. 
We should select the cluster heads and cluster members for the two clusters. Unlike the section 3.1.1 and 3.1.2, when the UAVs in set A and set B calculate the probability of them becoming the cluster head, they just consider the UAVs in the same set. Therefore, the special Hello packet is needed. Similarly, only the UAVs in previous cluster can join the two new clusters. This method decreases the ripple effect caused by the local re-clustering.

\section{Simulation results}

In simulation environment, some comparisons are made between CMPC algorithm and several mainstream clustering algorithms, which include MOBIC algorithm [1], DDVC algorithm [13], MPBC algorithm [12] and the clustering algorithm in MPCR algorithm [5]. The simulation software is NS-2(Network Simulator v2.34). IEEE 802.11 DCF [18] (Distributed Coordination Function) with CSMA/CA (Carrier Sense Multiple Access/Collision Avoidance) is used as the medium access control protocol. The carrier frequency of Hello packets is $600 \mathrm{MHz}$. We control the effective communication range of UAVs through changing the transmission power, the receiving sensitivity and the antenna gain. The channel adopts the free space attenuation model, the noise power is negligible. Other simulation parameters are given in Table 1.

Table 1 Simulation parameter

\begin{tabular}{cc}
\hline Parameters & Values \\
\hline Environment size & $50 \mathrm{Km} * 50 \mathrm{Km}$ \\
Number of UAVs & 100 \\
Transmitter range & $10 \mathrm{~km}-15 \mathrm{~km}$ \\
Mobility model & the model described in section 1 \\
& $40 \mathrm{~m} / \mathrm{s}-70 \mathrm{~m} / \mathrm{s}$ \\
Maximum speed of UAVs & Random \\
\hline UAV placement strategy &
\end{tabular}

Figure 3 shows the average link connection lifetime versus maximum movement speed for different algorithms when the maximum communication range is $10 \mathrm{~km}$. This performance index represents the average connection lifetime of links between the cluster head and its members. The longer the lifetime is, the better the performance of the clustering algorithm is. With the change of maximum movement speed, the average link connection lifetime of CMPC is longer than MPCR's, MPBC's, DDVC's and MOBIC's. That is because CMPC algorithm uses strict screening conditions in the process of cluster formation, which can increase the stability of the cluster head and its members. Therefore, the average link connection lifetime of CMPC algorithm is longest. At the same time, it indicates that CMPC algorithm is suitable for highly dynamic networks. All of the above algorithms' average link connection lifetime decreases with the maximum movement speed increases. That is because the increase of the movement speed results in more frequent change of network topology structure. Therefore, the average link connection lifetime decreases generally. The average link connection lifetime of DDVC algorithm declines more sharply than the other algorithms'. That is because DDVC algorithm is designed for pseudolinear mobile ad hoc network, DDVC algorithm is suitable for the mobility scenario that the change of movement speed is not frequent. 


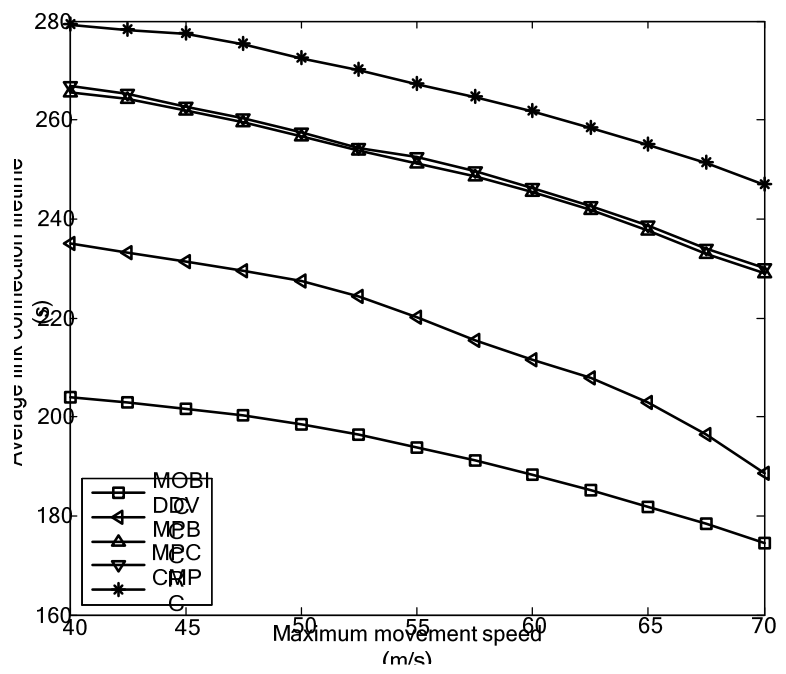

Fig. 3 Average link connection lifetime versus maximum movement speed

Figure 4 shows the average link connection lifetime versus maximum communication range for different algorithms when the maximum movement speed is $55 \mathrm{~m} / \mathrm{s}$. With the UAVs' maximum communication range increases, all of the above algorithms' average link connection lifetime increases. That is because the probability that cluster member moves out of the communication range of its cluster head decreases with the increase of maximum communication range. Therefore, the average link connection lifetime between the cluster head and its members increases. No matter how the UAVs' maximum communication range changes, CMPC algorithm can provide longer average link connection lifetime than the other algorithms.

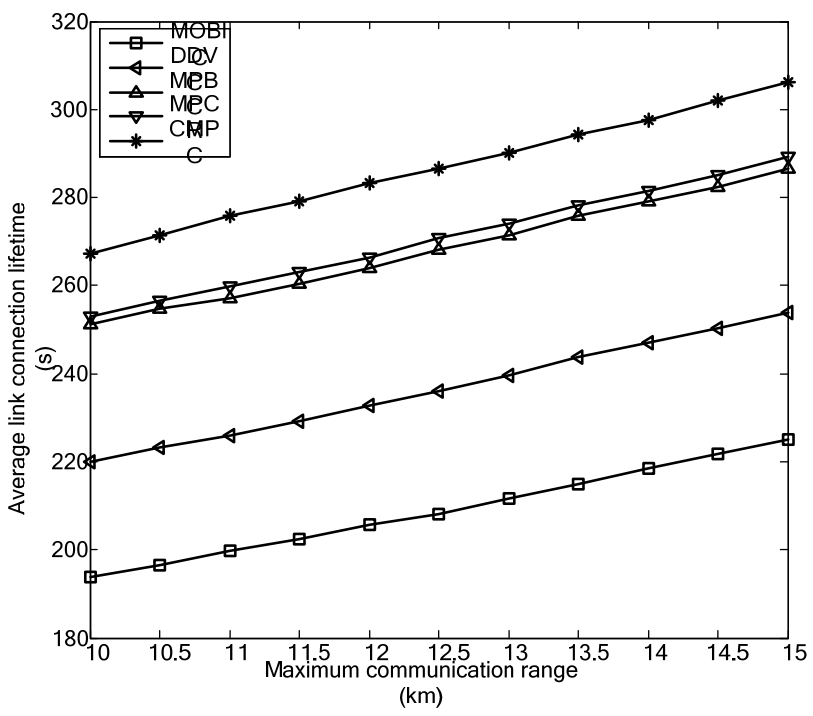

Fig. 4 Average link connection lifetime versus maximum communication range

Figure 5 shows the average cluster head lifetime versus maximum movement speed for different algorithms when the maximum communication range is $10 \mathrm{~km}$. With the maximum movement speed of UAVs increases, the network topology structure changes more dramatically, and the rotation of cluster heads is more frequent. Therefore, the average cluster head lifetime of the five clustering algorithms decreases with the movement speed increases. CMPC algorithm offers a strict scheme of selecting the cluster head. The cluster heads in CMPC algorithm have stronger stability. Therefore, the average cluster head lifetime of CMPC algorithm is longer than the other algorithms'. 


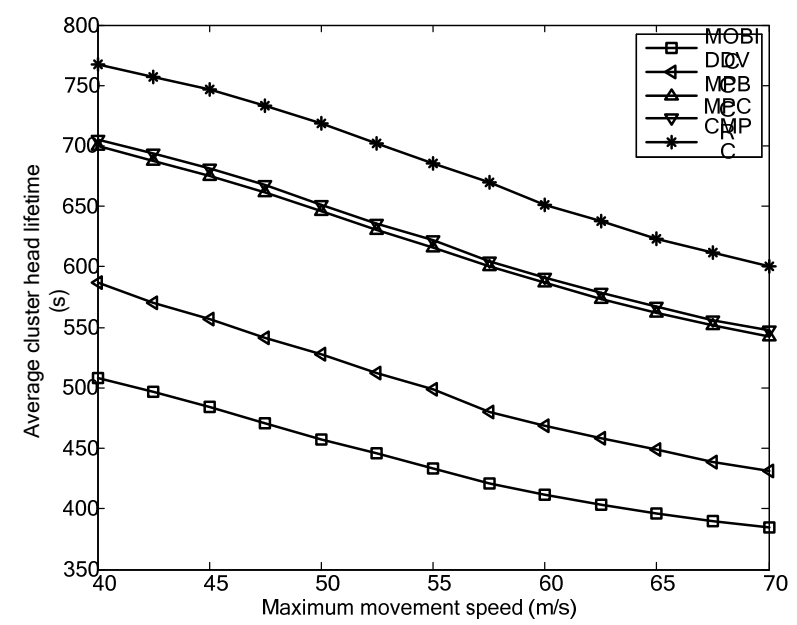

Fig. 5 Average cluster head lifetime versus maximum movement speed

Figure 6 shows the average cluster head lifetime versus maximum communication range for different algorithms when the maximum movement speed is $55 \mathrm{~m} / \mathrm{s}$. With the increase of UAVs' maximum communication range, the average cluster head lifetime of each algorithm increases. That is because the increase of communication range can dilute the effect of UAVs' high mobility, which can increase the stability of the cluster head. No matter how the UAVs' maximum communication range changes, the average cluster head lifetime of CMPC algorithm is longer than the other algorithms'.

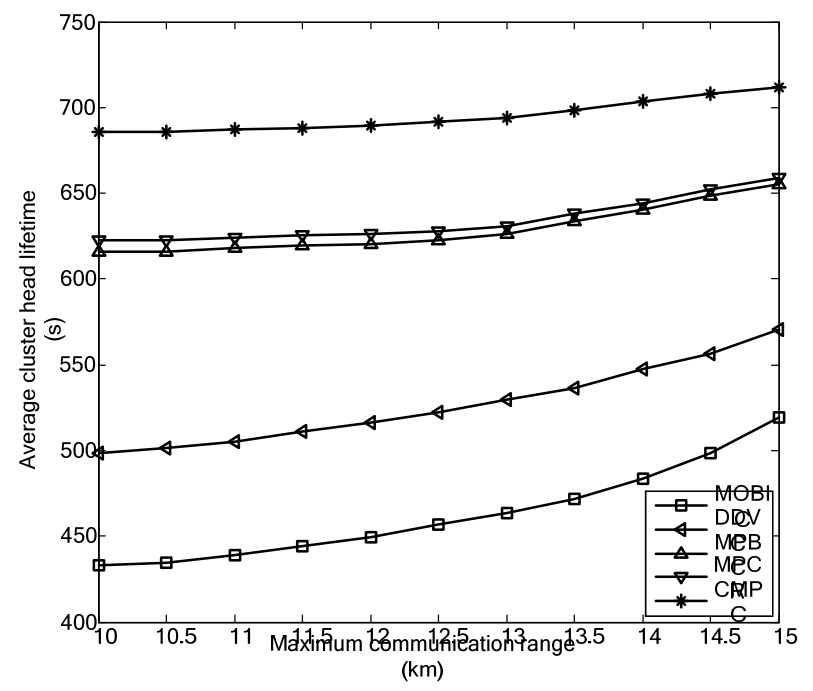

Fig. 6 Average cluster head lifetime versus maximum communication range

Figure 7 shows the average number of members per cluster versus maximum movement speed for different algorithms when the maximum communication range is $10 \mathrm{~km}$. No matter how the maximum movement speed changes, the average number of members per cluster of MOBIC, MPBC and MPCR is almost equal. When the maximum movement speed increases, the cluster member has a better chance of escaping from the communication range of the cluster head, so the number of UAVs in cluster decreases gradually. Therefore, the maximum movement speed and the number of members per cluster have negative correlation for the proposed five algorithms. The average number of members per cluster of CMPC is lower than the other algorithms. This is because CMPC has the strict rule of the members joining and leaving the cluster. In CMPC, not all UAVs in the cluster head's communication range can be selected as the cluster members, only satisfying some conditions can the UAVs be selected as the cluster members. At the same time, CMPC can predict the situation that some cluster member is about to leave the current cluster, and delete this cluster member beforehand 
in order to keep the cluster stable. However, this cluster member still belongs to the current cluster in the other algorithms.

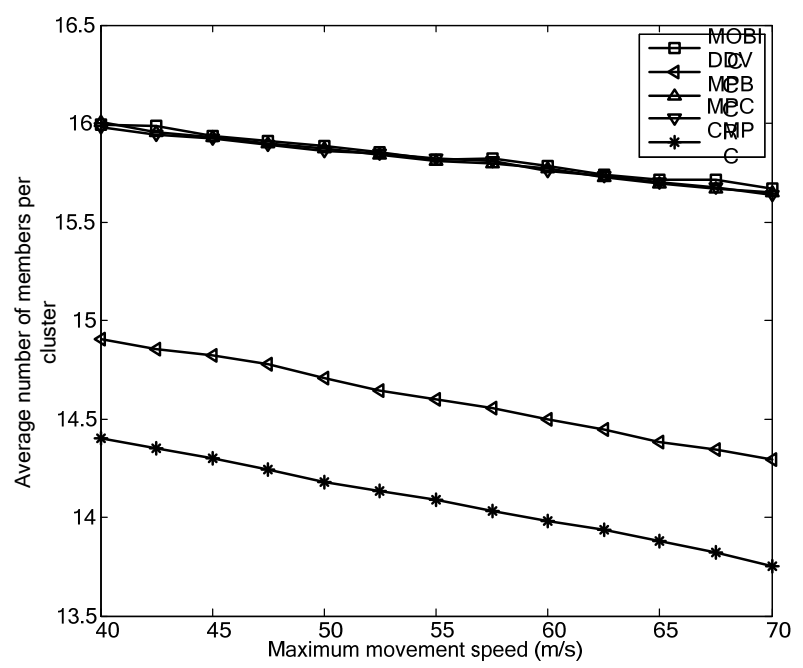

Fig. 7 Average number of members per cluster versus maximum movement speed

Figure 8 shows the average number of members per cluster versus maximum communication range for different algorithms when the maximum movement speed is $55 \mathrm{~m} / \mathrm{s}$. No matter how the maximum communication range changes the average number of members per cluster of MOBIC, MPBC and MPCR is almost equal. The average number of members per cluster of each algorithm increases with the increase of maximum communication range. That is because the number of one-hop neighboring UAVs of the current cluster head increases with the increase of maximum communication range. These UAVs are likely to be selected as the cluster members of the current cluster. CMPC algorithm still keeps the minimum average number of members per cluster.

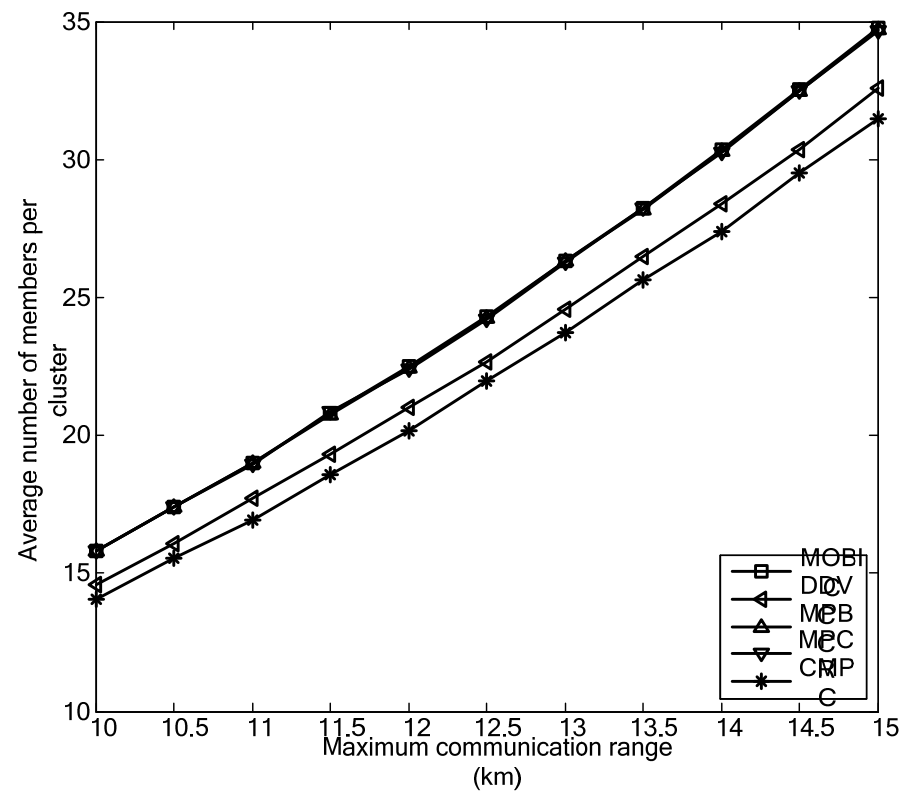

Fig. 8 Average number of members per cluster versus maximum communication range

\section{Conclusion}

If traditional clustering algorithms are adopted in highly dynamic large-scale ad hoc UAV networks, the performances would be so low that are unable to meet the demands. We propose a CMPC algorithm, which can realize cluster formation and maintenance in highly dynamic large-scale ad hoc UAV networks, and the established cluster structure is more stable, thus decreasing the control 
overhead which is caused by rebuilding the cluster. Simulated performance comparisons have been made among CMPC algorithm and MOBIC, MPBC, MPCR, DDVC algorithms, the results show that CMPC algorithm can ensure higher average link connection lifetime and cluster head lifetime, meanwhile, it indicates that CMPC algorithm can increase the stability of cluster structure. The algorithm we proposed is best for highly dynamic large-scale ad hoc UAV networks.

\section{References}

[1] P. Basu, N. Khan, T. Little. A mobility based metric for clustering in mobile ad hoc networks [C]// Proc. Distrib. Comput. Syst. Workshop,Mesa, AZ, Apr. 2001, pp. 413-418.

[2] F. Tolba, D. Magoni, P. Lorenz. Connectivity, energy and mobility driven clustering algorithm for mobile ad hoc networks [C] //Proc. IEEE GLOBECOM, Washington, DC, 2007, pp. 2786-2790.

[3] Q. Yu, N. Zhang, W. Meng, and F. Adachi. A novel stability weighted clustering algorithm for multi-hop packet radio virtual cellular network [C]// Proc. IEEE WCNC, Sydney, Australia, Apr. 2010, pp. 1-6.

[4] Y. Zhang and J. M. Ng. A distributed group mobility adaptive clustering algorithm for mobile ad hoc networks [C]// Proc. IEEE ICC, Beijing, China, May 2008, pp. 3161-3165.

[5] Jian Shu, Yangfan Ge, Linian Liu. Mobility Prediciton Clustering Routing in UAVs[C]/Proc of IEEE International Conference on Computer Science and Network Technology. 2011, pp. 1983-1987.

[6] Chunhua Zang, Shouhong Zang. Mobility Prediction Clustering Algorithm for UAV Networking [C]// Joint Workshop of SCPA 2011 and SaCoNAS 2011, IEEE, pp. 1158-1161.

[7] LIU Kesheng, ZHANG Jun and ZHANG Tao. The Clustering Algorithm of UAV Networking in Near-space [J]. IEEE, 2011, pp. 1550-1553.

[8] Mengqing Cai, Lanlan Rui, Danmei Liu. Group Mobility Based Clustering Algorithm for Mobile Ad Hoc Networks [J]. IEICE, 2015, pp. 340-343.

[9] Javad A. T, Mohammad R. M. A mobility-based cluster formation algorithm for wireless mobile ad-hoc networks [J]. Cluster Comput., 2011, pp. 311-324

[10] Nasser AL-QADAMI, Inas LAILA, et al. Mobility Adaptive Clustering Algorithm for Wireless Sensor Networks with Mobile Nodes [J]. ICACT, 2015, pp. 121-126.

[11]Xiaolin Tan, Zhongyang Xiong, Yun He. Signal attenuation-aware clustering in wireless mobile ad hoc networks [J]. Journal of Networks, 2013, 8(4), pp. 796-803.

[12] Minming Ni, Zhangdui Zhong, Dongmei Zhao. MPBC: A Mobility Prediction-Based Clustering Scheme for Ad Hoc Networks [J]. IEEE Transactions on vehicular technology, 2011, 60(9), pp. 4549-4559.

[13]Ehssan Sakhaee, Abbas Jamalipour. Stable clustering and communications in pseudolinear highly mobile ad hoc networks [J]. IEEE Transactions on vehicular technology, 2008, 57(6), pp. 3769-3777.

[14]J. M. Ng, Y. Zhang. A mobility model with group partitioning for wireless ad hoc networks $[C] /$ Proc of IEEE International Conference on Information Technology and Applications. 2005, pp.289-294.

[15] Andrea Goldsmith, Wireless Communication, Cambridge University Press, Cambridge, 2005.

[16] Csiszar V, Mori TF. A Bienayme-Chebyshev inequality for scale mixtures of the multivariate normal distribution[J]. Math Inequal Appl, 2009, 12(4), pp. 39-44. 
[17] S. Leng, Y. Zhang, H.-H. Chen, et al. A novel k-hop compound metric based clustering scheme for ad hoc wireless networks [J]. IEEE Trans. Wireless Commun, 2009, 8(1), pp. 367-375.

[18] IEEE Computer Society LAN MAN Standards Committee: Wireless LAN Medium Access Protocol (MAC) and Physical Layer (PHY) specification, IEEE Standard 802.11-1977. The Institute of Electrical and Electronics Engineers, New York (1997). 